\title{
Does IPE in a clinical environment lead to positive perceptions of interprofessional working?
}

Authors: Layla Fattah and Andrew Mawdsley, University of Manchester and Central Manchester Foundation Trust

Background: There is a current move to integrate inter-professional education (IPE) into the undergraduate curriculum at this institution. To support the IPE agenda, the Clinical Tutors developed and delivered a learning session for undergraduate medical and pharmacy students that engaged the students in "situated learning" in a clinical environment.

Description of work: Third year pharmacy and medical students assigned to placements at two local foundation trusts were recruited to attend a joint IPE teaching session. The aim of the session was to provide students with an opportunity to engage in authentic practice activities in a clinical environment adopting roles mirroring their respective roles in practice. The session focussed on the management of a real patient. Students were allocated to a clinical ward in small interprofessional groups of three or four. The student group interviewed a real patient to obtain a medication and drug history. They then used resources such as the BNF and NICE guidelines to review the patient's current medication and make suggestions for initiating drug treatment. Students were encouraged to write a prescription for the new items they intended to prescribe for the patient. This promoted collaborative decision making and situated learning. The session was intentionally semi-structured to allow students to naturally form team roles authentic to practice. All students completed a pre- and post-session evaluation utilising the validated IPE data collection tool, Readiness for Interprofessional Learning Scale (RIPLS) ${ }^{1}$.

Proposed evaluation: The session will be evaluated both quantitatively and qualitatively. RIPLS forms will be analysed using SPSS. Themes will be analysed from student written comments. The Clinical Tutors observed mixed dynamics in the group working processes and informally received both positive and negative feedback from the students. Focus groups will be undertaken to further explore the student experience of this IPE initiative.

\section{References}

1. Bligh J, Parsell G. Multi-professional working: learning from experience. Med Educ 1999;33(9):632-3. 